\title{
Profiling F/OSS Adoption Modes: An Interpretive Approach
}

\author{
David López ${ }^{1}$, Carmen de Pablos ${ }^{2}$, and Roberto Santos ${ }^{3}$ \\ 1 University of León, Spain \\ david.lopez@unileon.es \\ 2 Rey Juan Carlos University, Spain \\ carmen.depablos@urjc.es \\ 3 Telefónica de España, Spain \\ roberto.santossantos@telefonica.es
}

\begin{abstract}
This article presents the findings of a research aimed at characterizing F/OSS migration initiatives, in total 30 experiences have been considered, 19 of which have been conducted by public administrations and the rest by private firms, operating different industries in eight different countries.

Open source migration projects is a recent research topic, more so when considering it from a managerial perspective. To overcome the lack of theoretical models an empirical approach relying on grounded theory has been adopted as this inductive approach allows theory building and hypothesis formulation.

According to the results, migrating from proprietary into open source is dependent on contextual and organizational factors, as for example, the need of the change itself, the political support for the change, the suitability of IT, the organizational climate, the motivation of the human resources, the kind of leadership for the project or the firm complexity. Besides, migration efforts imply strategic and organizational consequences that the organization must evaluate well in advance.
\end{abstract}

\section{Introduction}

F/OSS as a radical approach to software development started in the early seventies consolidating itself as an alternative business model in the nineties. Since then F/OSS has been thoroughly studied from a technical perspective [1, 2] and as an emerging economic market [4, 5].

F/OSS is arguably a cost-effective solution in public administrations or education in which large investments in hardware are often required [3, 6] moreover the application of $\mathrm{F} / \mathrm{OSS}$ tools promotes innovation and industry development worldwide 7], 8, 9]. F/OSS migration initiatives are gaining momentum in the international arena as many developing countries are embracing this model for their ICT policies [10, [11].

Notwithstanding the benefits that F/OSS poses for administrations and large companies, there is still some reluctance among organizations. Migrating into 
F/OSS is, as Mr. Schiel technical leader at Munich city hall claims: "LiMux is not a technical project", he says. "Initially, the team approached the migration as a classical IT problem, but the real issues turned out to be different. "It's all about managing change for and with people." At this point we are able to confirm this quote as some of our interviewees do agree with Mr Schiel.

The purposes of this paper are twofold. In the first place presenting results regarding international $\mathrm{F}$ /OSS migration experiences. In the second place providing insights into open source adoption by organizations.

The latter goal is paramount to define adequate public policies aimed at fostering $\mathrm{F}$ /OSS adoption whereas the former goal provides practitioners with guidelines to maximize value in migration initiatives.

\section{Qualitative Inductive Research Approach}

Grounded theory belongs to the set of qualitative research methods aimed at developing theory grounded in existing data gathered from real scenarios [12]. Being grounded theory an inductive, discovery methodology it allows to produce emergent theory in areas in which there is still knowledge gaps.

Grounded theory is specially suited in context-based, process-oriented descriptions of organizational phenomena [13. Initially applied in psychology [14, [15] it has been successfully applied in the information systems area for some time now [17] including open source research [18].

Some scholars have studied technology adoption processes [19], 20] notwithstanding the usefulness of modeling user's behavior to technology adoption for our purposes a broader perspective is required. Adopting open source and subsequent migration of existing services is not a process conducted by a single person not even by a single business unit.

According to the above mentioned purposes of this study a microanalysis approach, that is organization centered, is mandatory. This approach departs from quantitative, positivist approaches [21, 22] in the sense that no previous hypothesis are formulated with regards to F/OSS adoption, on the contrary theory is being developed incrementally according to revealed data. We believe both micro -or firm level- and macro analysis complement each other reinforcing together into a global perspective of F/OSS.

\subsection{Data Collection}

In total 30 migration projects have been collected. Following Glaser and Strauss' technique of theoretical sampling, migration projects have been selected according to maximum variability in terms of context, size, purpose, ICT intensity among others. Having many several factors intervening at different intensity levels is key to define concepts, categories and relationships i.e emergent theory [15].

Nineteen of the total documented experiences correspond to public administrations from eight different countries (Brazil, France, Germany, UK, Spain, 
Finland) and ten for-profit organizations from small medium companies to large multinationals such as Audi or Peugeot. For every organization the following aspects have been initially considered:

1. Migration objectives.

2. Migration timeframe

3. Type of software or service migrated.

4. Migration cost.

5. Migration critical success factors.

6. Migration critical failure factors.

7. Migration outcome.

8. Perceived benefits.

Data collection in grounded theory is conducted iteratively 14 starting with a general exploratory character and later on more focused approaches towards relevant topics and structured interviews.

Whenever possible personal contact were established with project leaders in order to gather as much information as possible. This information was complemented with existing information in journals, magazines and technical reports in specialized repositories such as OSOR.eu or epractice.ed 1 .

Alongside this qualitative approach a survey among public administrations in Spain and Spanish software providers was conducted to further inquire into how market perceived open source as an alternative. Triangulating both qualitative and quantitative data provides more robust conclusions 12 .

\subsection{Data Analysis}

Inductive analysis aims at finding relevant concepts and relationships among them. Initially in the so called open coding researchers thoroughly analyze existing data and summarize it into categories [15]. In a second stage, axial coding, initial categories are further developed into subcategories representing variations along dimensions (i.e. axial coding), these subcategories often match questions such as: when, how, why, where and what for.

Given the intra-firm analysis perspective adopted in this research F/OSS migration experiences are considered as internal processes of change. We believe this process-centered approach reflects real organizations' structures therefore facilitating its adoption in existing business units.

As figure 1 shows, F/OSS migration projects are consisted of three main stages: -or main categories in Grounded theory parlance- Adoption Process, Migration Process and finally Migration Results. Then, for each category, several subcategories representing intervening factors are identified.

${ }^{1}$ Further information is available at:

http://cenatic.interoperabilidad.org:8080/web/guest/grandes-empresas

http://cenatic.interoperabilidad.org:8080/web/guest/

administraciones-publicas 


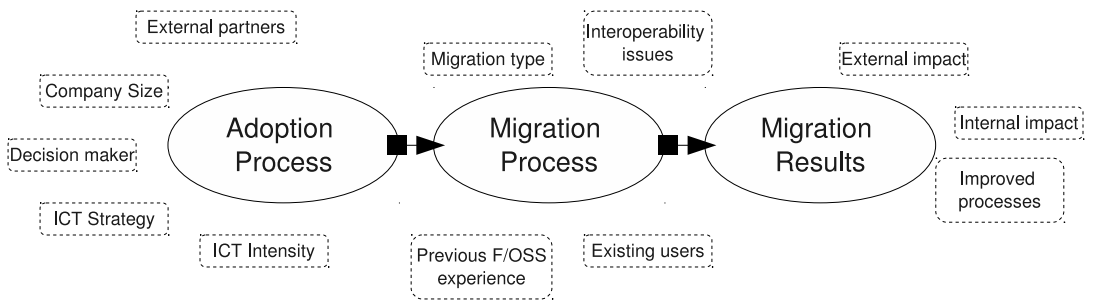

Fig. 1. F/OSS migration life cycle

\section{$3 \quad$ Research Results}

Three main modes of F/OSS adoption emerge from the analysis: Organizations concerned with vendor independence which consider open source software as a strategic asset. Organizations embracing open source as a better option than privative one in terms of performance or new features. Finally, in the third adoption mode, organizations striving for budget constrain consider open source as a cost-effective option in terms of licensing and hardware obsolesce.

First mode: Strategic movers. In this adoption mode, organizations have faced vendor issues such as forced migrations, ceasing product support or spiraling ICT costs. Removing vendor lock-ins is considered a priority by top executives who define strategic plans to evolve into the F/OSS paradigm.

These organizations tend to be large both in human resources and ICT budget. They have specific staff dedicated to ICT development and support.

Being intensive users of ICT services, for instance public administrations, they face interoperability issues with existing services as well as users' resistance to change established routines and procedures. They adopt a gradual approach to software migration usually with schedules in the order of years to complete. Sometimes rescheduling is mandatory.

Migration processes in this case often require external partners with technical expertise and large scale deployment experience specially if the organization has not previous experience or faces challenging scenarios of non-interoperable technologies and information.

The outcome of migration initiatives in this case are positive in general with positive results in terms of improved internal processes. Besides they usually create positive externalities in other business units. Due to the large scale nature of this initiatives often they impact society in general either by providing new services or improved software publicly available. Clear examples of this adoption mode are: Munich city hall, French police or USA postal service.

Second mode: Feature seekers. This category is consisted mainly of medium to large organizations with intensive use of ICT in their business processes looking for new opportunities to improve current existing IT capabilities, for instance supercomputing or embedded systems. Examples of this mode are Audi or Peugeot. 
Migration projects are initiated by internal experts taking advantage of recent developments, either software or hardware. An IT expert working for a financial institution claims that: "We are not fans of F/OSS we stick to the best solution given the context and existing options"

In spite of having technical expertise they usually collaborate with external partners for specific expertise. Due to the non-core nature of affected services interoperability is not a concern neither are end users who remain oblivious of backend reengineering programs.

Contrary to the previous category, migration timeframes remain within months with defined procedures well in advance. Success criteria are clearly determined. The results of this initiative are seldom transformative in the sense of producing impacts just on specific internal services.

Third mode: Budget optimizers. Small to medium companies often adopt open source as the best option in terms of price performance ratio. They usually implement new, non-core services, with open source as an inexpensive approach. The typical profile is a local administration or SME operating in a non-intensive ICT market.

\section{Implications for F/OSS Migration Processes}

As a general rule it seems a good practice to look for interoperable technologies in existing or future software deployments in order to ensure optimal vendor independence and flexibility to adapt to business requirements.

Some companies adopt a migration strategy relying on early adopters to test prototypes adapting them according to received feedback. We consider this approach quite adequate for it ensures business continuity while involving users from start, this reinforces the idea that F/OSS migration initiatives entail technical, organizational and business process reengineering [23].

According to our data most of the times large organizations hire external companies providing technical expertise or migration experience. Furthermore there exist some cases, mainly in very specialized areas such as mathematical modeling or embedded hardware, in which several partners collaborate to come up with an adequate solution.

No matter the F/OSS adoption mode-either strategic, functional or optimizingcompanies start considering open source as an alternative to specific issues and gradually have it adopted in subsequent initiatives. This behavior based on reinforced trust is consistent with technology acceptance models [19].

\section{Future Work}

F/OSS adoption offers good opportunities for reaching efficiency, flexibility and security in organizational processes, but it also poses challenging questions. Modeling users' response to technology changes is paramount to integrate new software into already existing organizations, at this point recent results enhance 
former Technology Acceptance Models (TAM) by considering software as a social actor within the organization able to interact with employees at increasing sophistication levels 20. We believe that further research could provide interesting results complementing this interaction-centric models with constructs valid at a firm and intrafirm level.

In every migration project there is always an internal sponsor or a group of people leading the initiative. Further research into leadership aspects of open source projects may serve practitioners to identify best organizational patterns to induce F/OSS adoption.

An interesting fact that emerged during the present research is that some organizations, mainly large multinationals, are able to generate positive externalities in other units or even contribute with their own developments back to the open source community. Being public administrations large ICT consumers providing further insight into effective means to induce innovation, software reutilization for instance, would be an interesting research topic.

There is international consensus on the importance that ICT has on education and development, 10 percent of broadband penetration increases GDP in developed economies up to 1.2 percent [24]. Being F/OSS capable of provisioning computers at lower costs there may be incentives by governments to engage in national initiatives to promote digital literacy relying on the F/OSS paradigm. Documenting exemplary initiatives in this matter is encouraged.

Acknowledgment. The authors gratefully acknowledge the support of CENATIC, the Spanish agency in charge of open source promotion and Telefónica.

\section{References}

1. Raymond: The Cathedral and the bazaar. Knowledge. Technology and Policy 12(3) (1999)

2. Hunter: Open Source Data Base Driven Web Development. Chandos, Oxford

3. Lerner, Tirole: The simple economics of Open Source. Journal of Industrial Economics 50(2), 197-234

4. Lerner, Tirole: The Economics of Technology Sharing: Open Source and Beyond. Journal of Economic Perspectives 19(2), 99-120

5. Riehle: The Economic Motivation of Open Source: Stakeholder Perspectives. IEEE Computer 40(4) (2007)

6. Lakhan, Jhunjhunwala: Open Source in Education. Educause Quarterly 31(2), $32-40$

7. Shiff: The Economics of Open Source Software: a survey of the early literature. Review of Network Economics 1(1), 66-74

8. Hippel, Krogh: Open source software and the private-collective innovation model: Issues for organization science. Organization Science 14(2), 209-223

9. Osterloh, Rota: Open source software development, just another case of collective invention. Research Policy 36(2), 157-171

10. Ahmed: Migrating from proprietary to Open Source: Learning Content Management Systems, Doctoral Dissertation, Department of Systems and Computer Engineering, Carleton University, Ottawa, Ontario, Canada 
11. UOC Report: The use of open source in Public Administrations in Spain, Universitat Oberta de Calalunya, Report

12. Myers: Qualitative Research in Business and Management. Sage, Thousand Oaks (2009)

13. Myers: Qualitative Research in information systems. MIS Quaterly 21(2), 241-242 (1997)

14. Glaser, Strauss: The discovery of Grounded Theory: Strategies for Qualitative Research. Aldine Publishing Company, New York (1967)

15. Strauss, Corbin: Basics of Qualitative Research: Grounded theory, Procedures and Techniques. Sage, Thousand Oaks (1990)

16. Orlikowski: Information Technology and the Structuring of Organizations. Research Approaches and Assumptions. Information Systems Research 2(1) (1991)

17. Orlikowski: CASE Tools as Organizational Change: Investigating Incremental and Radical Changes in Systems Development. MIS Quaterly (Septermber 1993)

18. Dedrick, West: Why Firms adopt Open Source Platforms: A grounded Theory of Innovation and Standards Adoption. MIS Quaterly. Special Issue on Standard Making (2005)

19. Venkatesh, V., et al.: User acceptance of information technology: Toward a unified view. MIS Quarterly 27(3) (2003)

20. Sameh, Izak: The Adoption and Use of IT Artifacts: A New Interaction-Centric Model for the Study of User-Artifact Relationships. Journal of the Association for Information Systems 10662(9) (2009)

21. Gonzalez-Barahona: About free Software, Rey Juan Carlos University-Dykinson (2004)

22. Wheeler: Why Open Source Software. Look at the Numbers

23. Hammer: Reengineering Work: Do not Automate, Obliterate. Harvard Business Review (2000)

24. Berkman Center: Berkman Center for internet and society. Broadband study for FCC (2009) 